\title{
Octave-Spanning Broadband Absorption of Terahertz Light Using Metasurface Fractal-Cross Absorbers
}

\author{
Mitchell Kenney,*® James Grant, Yash D. Shah,® Ivonne Escorcia-Carranza, Mark Humphreys, \\ and David R. S. Cumming*
}

School of Engineering, University of Glasgow, Glasgow, G12 8LT, United Kingdom

Supporting Information

ABSTRACT: Synthetic fractals inherently carry spatially encoded frequency information that renders them as an ideal candidate for broadband optical structures. Nowhere is this more true than in the terahertz $(\mathrm{THz})$ band where there is a lack of naturally occurring materials with valuable optical properties. One example are perfect absorbers that are a direct step toward the development of highly sought after detectors and sensing devices. Metasurface absorbers that can be used to substitute for natural materials suffer from poor broadband performance, while those with high absorption and broadband capability typically involve complex fabrication and design and are multilayered. Here, we demonstrate a polarization-insensitive ultrathin $(\sim \lambda / 6)$ planar metasurface $\mathrm{THz}$ absorber composed of supercells of fractal crosses capable of spanning one optical octave in bandwidth, while still being highly efficient. A sufficiently thick polyimide interlayer produces a unique absorption mechanism based on Salisbury screen and antireflection responses, which lends to the broadband operation. Experimental peak absorption exceeds

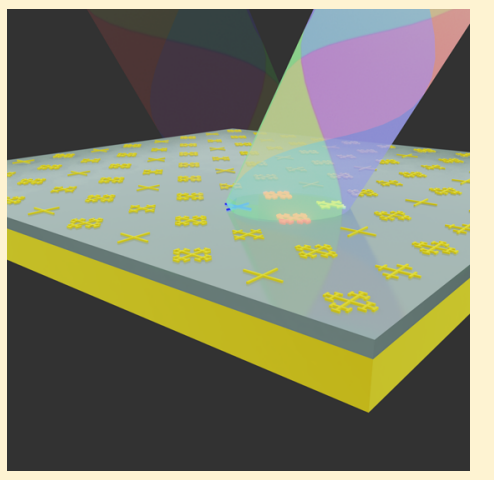
93\%, while the average absorption is $83 \%$ from $2.82 \mathrm{THz}$ to $5.15 \mathrm{THz}$. This new ultrathin device architecture, achieving an absorption-bandwidth of one optical octave, demonstrates a major advance toward a synthetic metasurface blackbody absorber in the $\mathrm{THz}$ band.

KEYWORDS: metasurface, metamaterial absorber, absorber, terahertz absorber, broadband absorption, fractal

$\mathrm{T}$ erahertz $(\mathrm{THz})$ radiation is typically understood to be the frequency range between 0.1 and $10 \mathrm{THz}$. Advancements in $\mathrm{THz}$ science and technology have resulted in several important applications including medical and security imaging, nondestructive testing, explosive detection, and wireless communications. Research has been driven by the unique properties of $\mathrm{THz}$ radiation: it is nonionizing thus safe to biological tissue; transparent to several common plastics and fibers; and has a shorter wavelength than millimeter waves, giving a higher spatial resolution. In addition several materials, such as explosives and illicit drugs, have characteristic $\mathrm{THz}$ spectroscopic signatures that can be readily identified. ${ }^{1-6}$ Despite a great deal of research into developing $\mathrm{THz}$ optical components, there still exists a need for high-efficiency and broadband devices for implementation in practical applications.

Optics research in the past decade has focused on the investigation into $2 \mathrm{D}$ planar metamaterials, called metasurfaces, where many recent useful applications and phenomena have been exhibited, including flat lenses, ${ }^{7-9}$ holograms, ${ }^{10,11}$ beam deflectors, ${ }^{12,13}$ chiral optics (chiroptics), ${ }^{14-16}$ and cloaking. ${ }^{17-19}$ These metasurfaces have the potential to synthetically overcome the poor interaction of natural materials with $\mathrm{THz}$ radiation. One such device is the perfect $\mathrm{THz}$ blackbody absorber wherein all light is perfectly absorbed at $\mathrm{THz}$ frequencies. Great progress toward developing a perfect metasurface absorber has been made in recent years, ${ }^{20-23}$ and while high peak absorption has been achieved, the bandwidth has only been very narrow $(Q>5)$.

Typically, the strong absorbers reported to date use a metalinsulator-metal (MIM) structure where the top layer is comprised of metallic resonant elements separated from the metal ground-plane by a dielectric interlayer, akin to the traditional Salisbury Screen. ${ }^{24,25}$ Owing to the distance between the two metal layers a magnetic dipole can be produced in the dielectric spacer that is formed by the resonant currents arising in the metal layers. The dipole in turn allows tuning of the magnetic permeability $\mu$ of the device such that the device impedance $Z=\sqrt{\mu / \varepsilon}$ can be closely matched to the free-space impedance $Z_{0}=377 \Omega$, resulting in a large absorption of the incident light. ${ }^{20}$ Since these structures rely on a strongly resonant phenomenon, they only achieve high absorption for very narrow bandwidth. Designs that have overcome this problem stack layers of resonators and dielectrics, ${ }^{26-32}$ related to the classical Jaumann absorbers. ${ }^{25}$ The main issue with the multilayer approach is that such devices involve very complex designs and fabrication procedures in which multiple layers lead to these absorbers being thick and bulky, hence, difficult to integrate with commercial technologies, as well as costly to manufacture. An alternative to using multilayer stacks of metal

Received: August 12, 2017

Published: September 6, 2017 
(a)

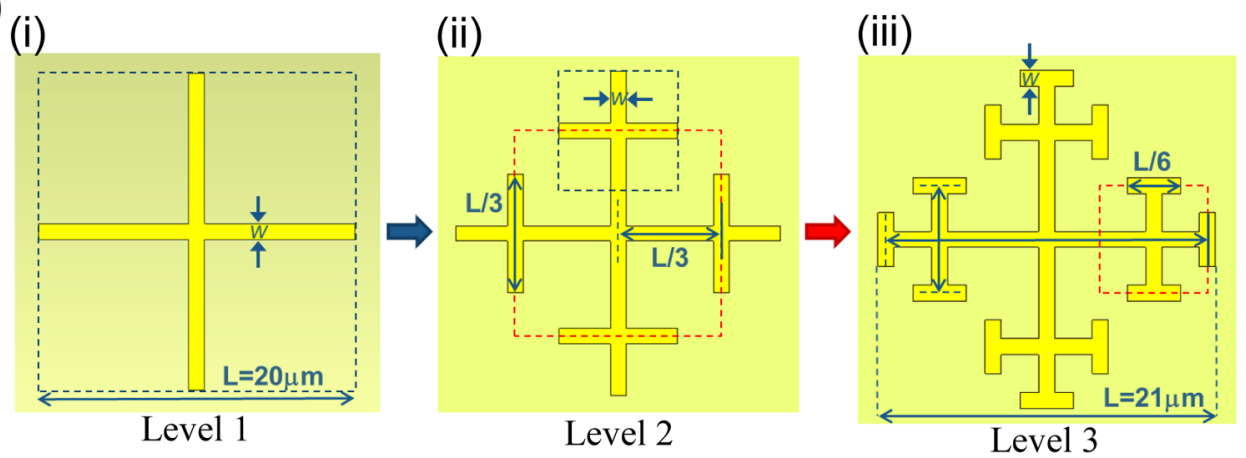

(b)

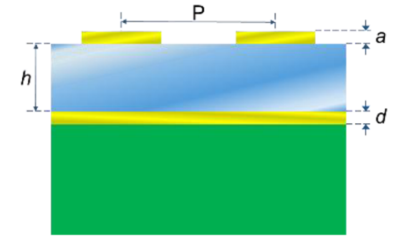

(d)

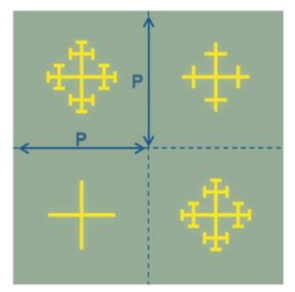

(c)

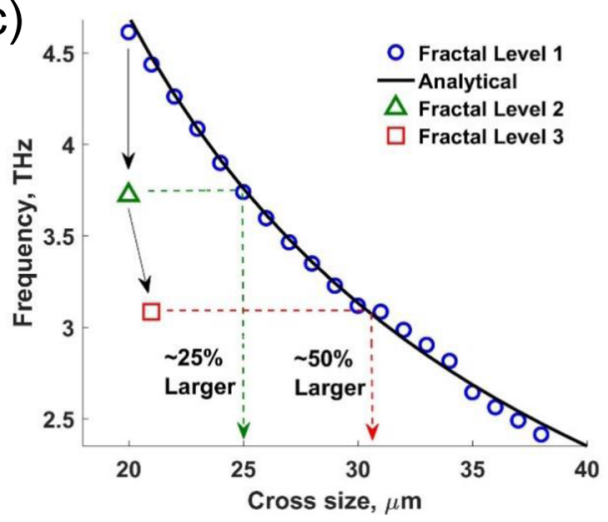

Figure 1. Fractal supercell absorbers. (a) Modification of a simple cross by increasing the fractal order, labeled as (i) fractal level 1, (ii) fractal level 2, and (iii) fractal level 3. Fractal levels 1 and 2 length $L=20 \mu \mathrm{m}$, level 3 length $L=21 \mu \mathrm{m}(0.5 \mu \mathrm{m}$ longer on each end) due to the addition of the smaller caps. Line width $w=1 \mu \mathrm{m}$ for all structures. (b) Side-view of the absorbing metasurface design, comprised of metal-insulator-metal; $a=d=$ $100 \mathrm{~nm}, h=5 \mu \mathrm{m}$ or $11 \mu \mathrm{m}$. (c) Plot of the simulated and analytical resonant absorption frequency vs cross size for level 1 fractal. Higher order fractals can be compared to larger simple crosses. (d) Overview of the supercell formed by a combination of fractal levels $1-3$. Period $P=40 \mu \mathrm{m}$, supercell period $=80 \mu \mathrm{m}$.

is to make a planar arrangement and combine multiple resonant elements with different resonant frequencies to form a metasurface. ${ }^{26,33}$ The planar absorber approach is better suited to the development of practical components due to its ultrathin design and fewer processing steps. However, devices that have been presented thus far have relied on a trade-off between peak absorption and maximum bandwidth, hence imperfections are inherent to these designs. ${ }^{26,34}$

An alternative approach to the scaling of the resonant elements to achieve a resonance frequency shift is to increase the effective perimeter of the element; this is achieved by incorporating a fractal design, where self-similar shapes are scaled down in size and added onto the original shape. ${ }^{35,36}$ This self-similarity can modify the effective inductances and capacitances of the resonant element, leading to a red-shift in the resonant frequency with increasing fractal order. In essence, an increase of the fractal order of a resonator is equivalent to increasing the effective electric length of the fundamental resonator, where only the fill-factor changes and not the size. Therefore, fractals offer a new degree of freedom, independent of the conventional scaling rule of resonator size, to achieve a variation in resonance frequency. In doing so, fractal resonators can remain much more subwavelength and compact in size. Work on fractal resonance has been particularly useful for antenna design and applied to various frequency regimes for applications in metamaterials. ${ }^{37,38}$ However, few studies have been carried out on the application of fractal structures for use in absorbers ${ }^{39-41}$ and scarcely any have investigated the $\mathrm{THz}$ regime where no natural material is available. Work carried out by Yang et al. in the visible band (ref 40) used a pseudofractal planar metamaterial design combined with amorphous silicon (a-Si) to obtain a very broadband absorption response. However, this result is mainly attributed to the classical p-i-n junction absorption by a-Si as opposed to a metamaterialdominant response, where the metamaterial only improved absorption by $10-20 \%$. Therefore, finding a broadband absorber for use in applications, which is not totally reliant on the bulk material absorption properties and, more so, on the electromagnetic effects by a metasurface, is still needed.

Here, we investigate the use of fractal resonators for octavespanning broadband absorption in the $\mathrm{THz}$ band. By combining structures of varying fractal orders into supercell arrangements, we are able to broaden the Full-Width at HalfMaximum (fwhm) bandwidth to exceed $45 \%$ absorption over one optical octave in frequency, from 2.75 to $5.56 \mathrm{THz}$. An experimental peak absorption of $93 \%$ is obtained, and we show that individual fractal resonances are broadened without loss of performance by increasing the dielectric spacer thickness, owing to an interference-dependent absorption effect distinct from many typical metamaterial absorbers. Additionally, we calculate the average peak absorption of the device to be $83 \%$ (from 2.82 to $5.15 \mathrm{THz}$ ). Our device is intrinsically polarization-insensitive due to the 4-fold symmetric arrangement and is expected to perform robustly at large incident angles without a significant loss of absorption. This highly absorbing metasurface covering an octave in bandwidth demonstrates the best performance of 
$5 \mu \mathrm{m}$
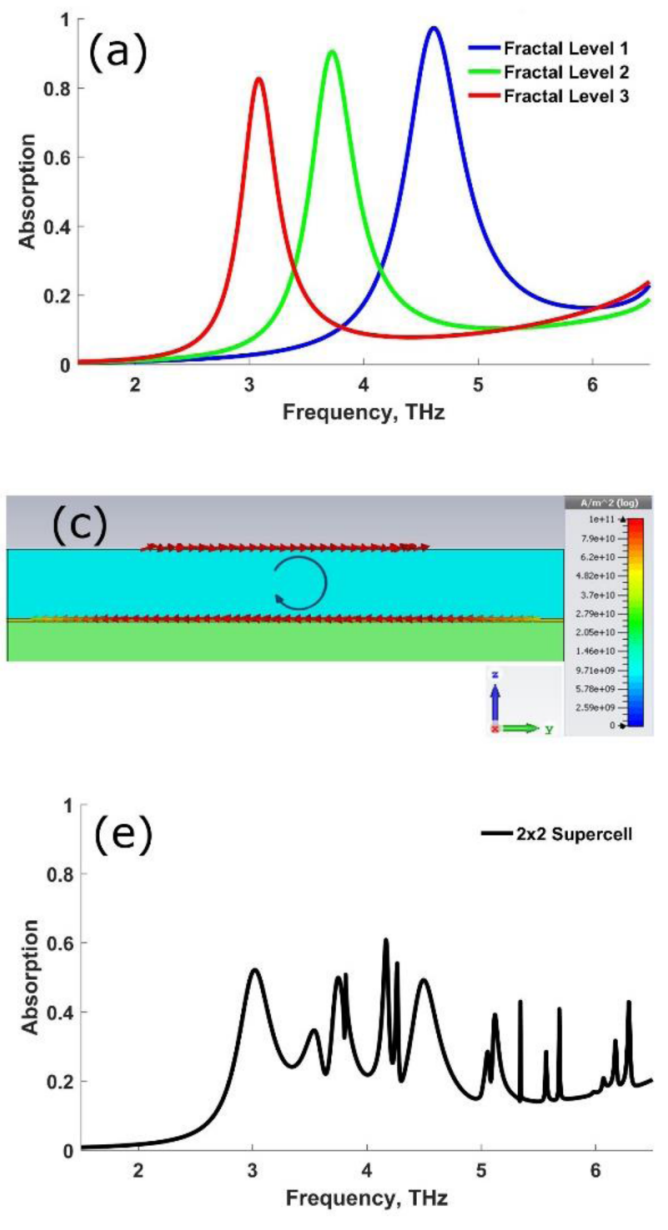

$11 \mu \mathrm{m}$
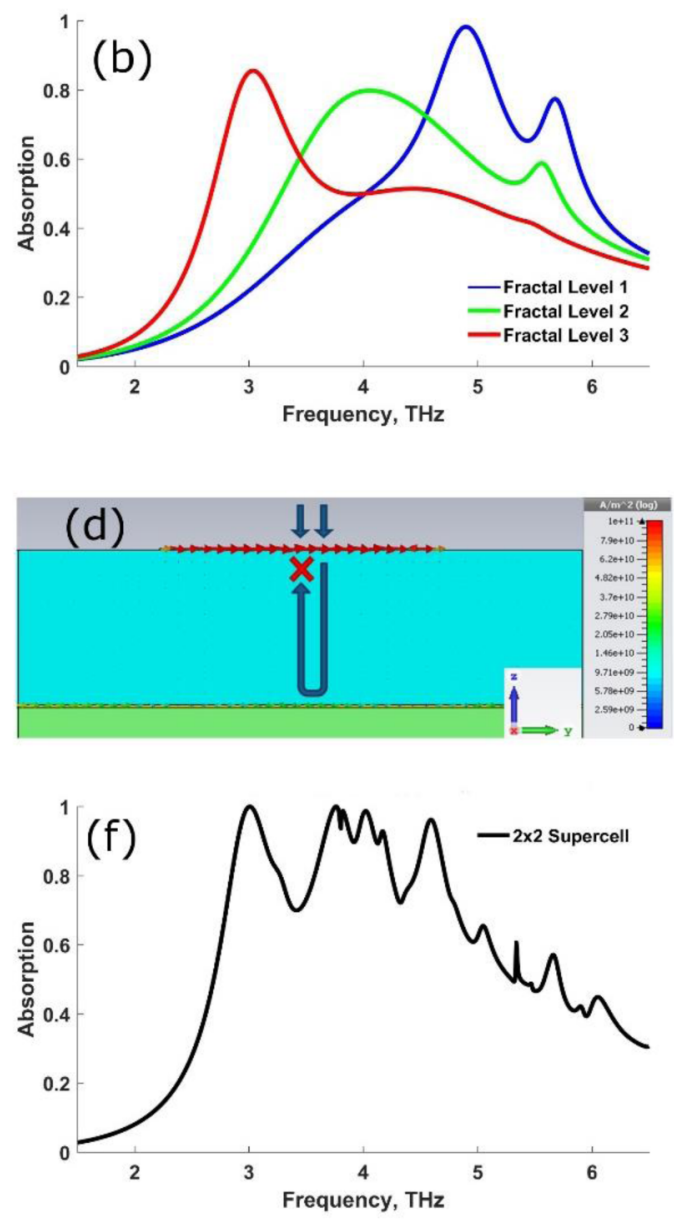

Figure 2. Simulations of various fractal absorber designs. (a), (c), and (e) (left column) are for $5 \mu$ m thick polyimide, while (b), (d), and (f) (right column) are for $11 \mu \mathrm{m}$ thick polyimide. (a, b) CST simulations for single fractal designs, showing (a) narrowband absorption and (b) broadband absorption. (c, d) 2D current density plots showing the current flows at resonance for the level 2 fractal design. (c) For $5 \mu \mathrm{m}$ thick polyimide, currents flow on both the fractal cross and ground-plane in opposite directions, forming an effective current loop. (d) For $11 \mu \mathrm{m}$ thick polyimide, current flow is only present on the fractal cross. (e, f) CST simulation of the broadband supercell arrangement for (e) $5 \mu \mathrm{m}$ polyimide and (f) $11 \mu \mathrm{m}$ polyimide thicknesses. Light was normally incident for all simulations.

any planar broadband $\mathrm{THz}$ absorber to date and paves the way toward ideal blackbody absorbers and sensors in the $\mathrm{THz}$ regime.

\section{RESULTS AND DISCUSSION}

The metal cross resonator (Figure 1a,i) is well-known for its use in plasmonic and metamaterial devices because of its polarization-insensitive and frequency-scalable nature. ${ }^{42}$ One such device is the MIM absorber (Figure 1b), where a dielectric interlayer of thickness $h$ is optimized to control the absorption response. By choosing a periodicity $P=40 \mu \mathrm{m}$, dielectric thickness $h=5 \mu \mathrm{m}$, a cross width $w=1 \mu \mathrm{m}$, and length $L=20$ $\mu \mathrm{m}$, we simulated the absorption frequency shift when $L$ is increased (Figure 1c). We settled at $40 \mu \mathrm{m}$ due to the trade-off between minimal coupling and maximized broadband absorption performance (see Supporting Information, note 6). It is evident that the resonance frequency (the frequency position of maximum absorption), represented by the blue circles, shifts to lower frequencies with increasing cross length, as expected. Due to this inverse relationship between resonance frequency $(f)$ and cross length $(L)$, we formulated the simple analytical equation $f=G \frac{c}{L n}$, where $c=3 \times 10^{8} \mathrm{~ms}^{-1}$ is the speed of light in a vacuum, $n$ is the refractive index of the dielectric interlayer, and $G$ is a coupling coefficient found by fitting to be 1.9. In our design we used polyimide PI2545 as the dielectric interlayer with a refractive index of $n=1.68+0.06 i .^{26,34}$ We used gold $\mathrm{Au})$ for the crosses and ground-plane, with thicknesses $a=d=$ $100 \mathrm{~nm}$ (Figure 1b) and, assuming that there is no loss in the $\mathrm{THz}$ regime, a frequency-independent conductivity of $\sigma=4 \times$ $10^{7} \mathrm{Sm}^{-1} \cdot{ }^{26,34}$ It is clear that the analytical curve (black solid line in Figure 1c) matches the simulation results well, which allows us to determine the resonant frequency of any arbitrarily chosen level 1 cross size.

To incorporate fractal properties into our design, two choices of self-similarity were selected. The first increase in fractal order was carried out by scaling the level 1 cross by one-third $(L / 3)$ and translating it to the ends of the level 1 cross arms (Figure 1a,ii). The blue dashed box around Figure 1a,i corresponds to the inclusion into the level 2 fractal in Figure 1a,ii (smaller blue square). The absorption peak occurs at $4.61 \mathrm{THz}$ for the level 1 
fractal cross ( $5 \mu \mathrm{m}$ polyimide thickness), while the level 2 fractal cross has its resonance absorption red-shifted to 3.72 $\mathrm{THz}$, as indicated by the green triangle in Figure 1c. This increase of the fractal level yielded a resonance frequency shift equivalent to a $\sim 25 \%$ size increase of the level 1 fractal (dotted green line, Figure 1c). Significantly, this red-shifting was achieved without an increase of the cross length. The level 3 fractal cross (Figure 1a,iii) was obtained by shrinking a region of the level 2 fractal cross (red dashed box, Figure 1a,ii) by $1 / 2$ and translating it to the ends of the cross arms. The extra "caps" on the end of the level 3 fractal cross are of length $L / 6$. The level 3 cross size is slightly increased to $21 \mu \mathrm{m}$ due to the extra $0.5 \mu \mathrm{m}$ on each end, with resonance occurring at $3.08 \mathrm{THz}$ (red square, Figure 1c). This frequency shift corresponds to a $\sim 50 \%$ equivalent size increase of a level 1 cross (dotted red line, Figure 1c). All crosses have a fixed width of $w=1 \mu \mathrm{m}$, unchanged with the aforementioned scaling.

An important step in the design of an MIM absorber is choosing the necessary dielectric thickness to tailor the absorption magnitude. To this end, a range of dielectric thicknesses were investigated for each fractal cross design. Numerical simulations were carried out at normal incidence using the commercially available CST Microwave Studio software (see Methods). A range of polyimide thicknesses from $3 \mu \mathrm{m}$ up to $13 \mu \mathrm{m}$ were investigated. Results for all simulations are given in Supporting Information, note 1. The two polyimide thicknesses with the best responses for singleband and broad-band operation were 5 and $11 \mu \mathrm{m}$, respectively. Simulation results for these thicknesses are shown in Figure 2. The absorption spectra for individual fractal levels 1-3 are shown in Figure $2 \mathrm{a}, \mathrm{b}$ for 5 and $11 \mu \mathrm{m}$ polyimides, respectively. It is clear that for $5 \mu \mathrm{m}$ polyimide (Figure $2 \mathrm{a}$ ), a narrow-band absorption occurs for each fractal cross, with absorption exceeding $80 \%$ for all designs. A slight decrease of the absorption efficiency takes place from fractal cross level 1 (97\%) to level 3 (83\%), best explained by the frequency shift no longer corresponding to optimal impedance matching due to reduced electromagnetic coupling between the cross and the ground-plane. Resonance frequencies (and absorption magnitudes) for fractal levels $1-3$ are $4.61 \mathrm{THz}(97 \%$ abs.), $3.72 \mathrm{THz}$ (90\% abs.), and $3.08 \mathrm{THz}$ ( $83 \%$ abs.), respectively. To investigate the physical process taking place for the large narrow-band absorption, 2D current-density plots were numerically simulated for the level 2 fractal cross structure (Figure 2c). The plot shows currents both on the fractal cross and the ground-plane. The opposing currents form an effective current loop, indicated by the circulating arrow inset in Figure $2 c$, well-known in metamaterial absorbers. ${ }^{43}$ Also, it is seen that the induced ground-plane current extends beyond the dimensions of the cross and, therefore, potentially undergoes a coupled dipolar resonance to the neighboring ground-plane currents. Increasing the dielectric thickness from 5 to $11 \mu \mathrm{m}$ yields little change in absorption magnitude for the single-band absorbers (Figure 2b), and the resonance frequencies are shifted slightly, with peak absorption for fractal levels 1-3 occurring at 4.90, 4.06, and $3.03 \mathrm{THz}$ (with corresponding absorption magnitudes of $98 \%, 80 \%$, and $85 \%$, respectively). However, a drastic change in the line width of each absorber is evident, with the bandwidth becoming extremely broadband. Such a result seems counterintuitive to the established explanation, where the absorption takes place due to a strong induced current loop between the cross and the ground-plane. The resonance frequency of the level 2 fractal cross is $\sim 4 \mathrm{THz}$ (which corresponds to a wavelength of $\lambda_{0}=75 \mu \mathrm{m}$ in free space), which within the dielectric layer is $\lambda_{0}=\lambda_{0} / n=45 \mu \mathrm{m}$ (where $n \approx 1.68$ ). We can therefore assume "Salisbury screen" and antireflection (AR) coating type operation, where the dielectric thickness of $11 \mu \mathrm{m}$ is then adequately $\sim \lambda / 4$, as required. This implies that the absorption process taking place is no longer due to EM coupling but instead wave interference. To confirm this, $2 \mathrm{D}$ current density plots were simulated for the level 2 fractal cross with an $11 \mu \mathrm{m}$ polyimide spacer (Figure $2 \mathrm{~d}$ ). Strong current flow is present only on the cross, with very diminished current flow on the ground-plane. It is assumed that the typical absorption mechanism ${ }^{20,42}$ does not occur, and the absorption phenomena for much thicker samples is due to a wave-interference mechanism, where destructive interference of the incident and reflected light results in minimal reflection and maximal absorption.

By combining multiple fractal crosses of different orders into one design, we aim to cover absorption over a broad frequency spectrum. We arranged the three fractal crosses into a supercell arrangement, as shown in Figure 1d. This arrangement was chosen, as it is 4-fold symmetric in both rotation and mirroring, therefore, imparting polarization insensitivity. This was verified using numerical simulations for both $5 \mu \mathrm{m}$ (Figure 2e) polyimide and $11 \mu \mathrm{m}$ (Figure 2f) polyimide thicknesses. Although both spectra show broadband operation, there is a substantial reduction in the absorption efficiency for the $5 \mu \mathrm{m}$ sample. This can be explained by the fact that each fractal cross level has a corresponding phase delay with respect to the incident light. For single band designs (Figure 2a), each cross is in phase with its neighbor upon resonance and, therefore, provides equal responses for cross-to-ground-plane coupling resulting in coherent induced magnetic resonance and high absorption. However, when combining multiple crosses into a supercell arrangement, the differences in phase will manifest in a hybridization of the ground-plane excitations. As seen in Figure $2 c$, the ground-plane currents extend almost to the edge of the unit cell. Therefore, with multiple crosses arranged into a supercell, we will no longer obtain a coherent excitation of induced magnetic dipoles, thus, leading to a decrease in the absorption capability. Because each cross has its absorption resonance occurring at different frequencies, it is not possible to numerically simulate for the $2 \mathrm{D}$ current density plots over a broad bandwidth to excite all crosses at the same time. Therefore, we cannot see the current densities from each cross resonance present on the ground-plane simultaneously. Instead, current density plots at each single-band resonant frequency, and the supercell broadband design also at these frequencies are given in the Supporting Information, note 5. The results show diminished ground-plane currents in the supercell design, thereby leading to a reduction in absorption.

The results for the $11 \mu \mathrm{m}$ thick polyimide sample (Figure 2f) clearly shows broadband operation, while simultaneously exhibiting strong absorption of light. Peak absorption magnitude exceeds $95 \%$ at three frequencies, with a minima of $70 \%$ in this broadband range. We calculate the average absorption to be $86 \%$, defined by the mean spectra value above the $70 \%$ minima. This calculation is further detailed in the Supporting Information Note 2. The fwhm bandwidth is $\Delta f=$ 2.61 THz between $2.69 \mathrm{THz}$ and $5.30 \mathrm{THz}$, which is centered at $f_{\mathrm{c}}=3.99 \mathrm{THz}$. We can calculate the fractional bandwidth of the device, defined as $\Delta f / f_{\mathcal{c}}$ which equates to $\sim 65 \%$; this is a very significant value for such a planar $\mathrm{THz}$ absorber, previously only achieved using complex multilayer designs. Additionally, 

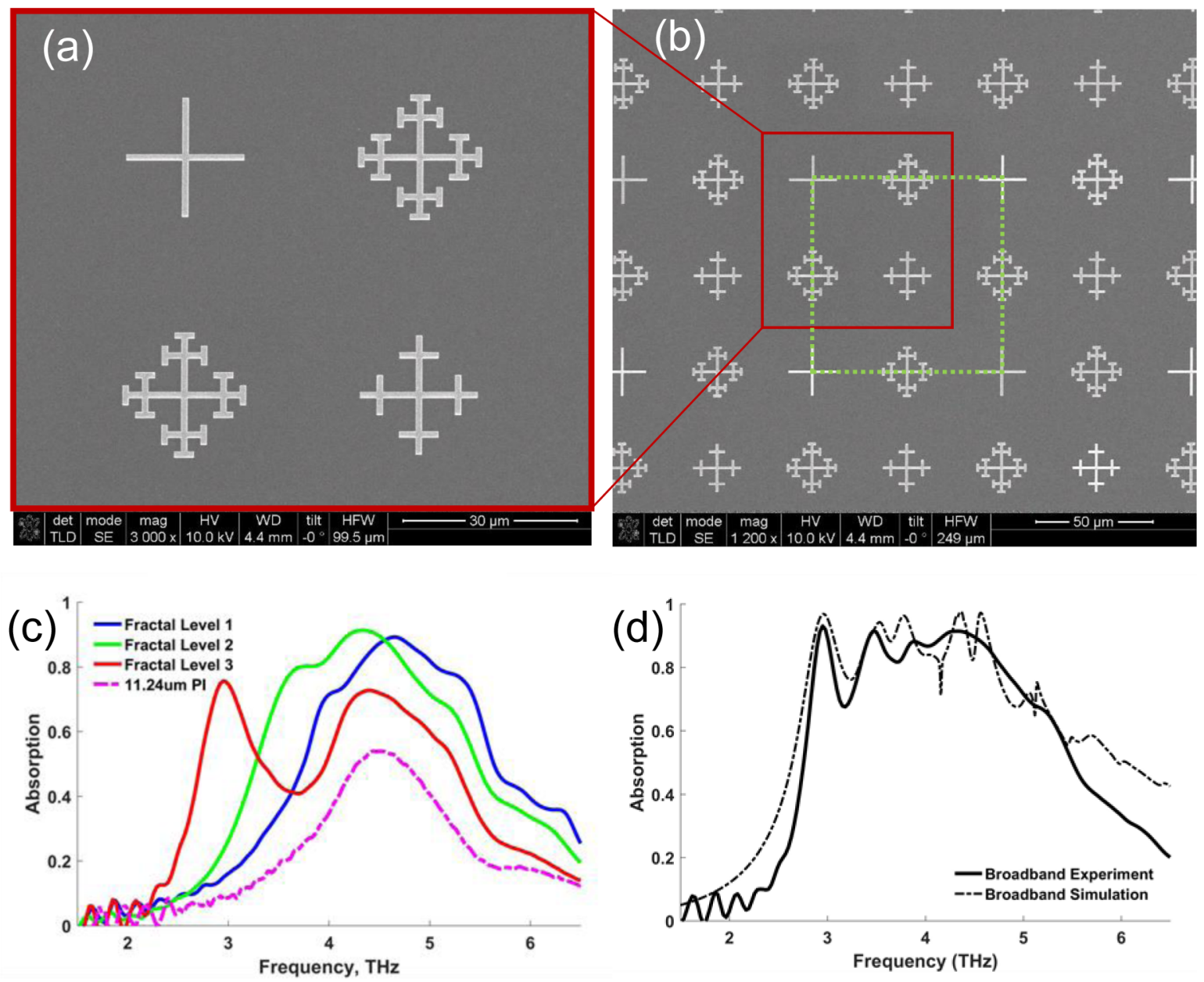

Figure 3. Scanning electron micrographs and experimental results: (a) Micrograph of a supercell used for the broadband absorption design. Supercell size $80 \mu \mathrm{m} \times 80 \mu \mathrm{m}$. (b) Zoomed-out micrograph. The green dotted box shows that the design has 4-fold mirror and rotational symmetry, hence insensitive to polarization. (c) FTIR absorption spectra for individual fractal designs. The magenta curve shows the absorption of polyimide alone. (d) Both experimental and simulated results for the supercell design, showing high-absorption broadband operation.

the device bandwidth is almost equivalent to one optical octave, where the fwhm bandwidth range is such that the maximum frequency $\left(f_{\max }=5.30 \mathrm{THz}\right)$ is a factor of 2 larger than the minima $\left(f_{\min }=2.69 \mathrm{THz}\right)$, giving a slightly smaller value of $f_{\max } / f_{\min }=1.97$. Therefore, the broadband performance of this design is superior to any other planar $\mathrm{THz}$ absorber to date, which are comparatively narrowband and have very small fractional bandwidths. As a note of interest to the reader, whereby we previously investigated the equivalence of levels 2 and 3 fractal crosses to simple scaled crosses (Figure 1c), we performed simulations to demonstrate the case for replacing the supercell of fractal crosses for a supercell of scaled simple crosses. The results are given in Supporting Information, note 6. Results show that the simple crosses perform similarly to the fractal crosses and provide a broad absorption bandwidth, yet show extremely strong cross-to-cross coupling for the simple cross case, which does not occur for the supercell design with the fractal crosses.

To experimentally verify our designs, fractal cross samples were fabricated based on both 5 and $11 \mu \mathrm{m}$ polyimide thicknesses using conventional lift-off processes (details given in Methods). Due to the tolerances of the spin-coating procedure for polyimide, the obtained average thicknesses were instead 5.59 and $11.24 \mu \mathrm{m}$, as measured by a Dektak profilometer, rather than the simulated 5 and $11 \mu \mathrm{m}$ values. For each polyimide thickness, four samples were fabricated: three singleband devices, consisting of the three fractal cross levels, and one broadband device based on the supercell design. Scanning electron microscopy (SEM) was used to image the devices, where a thin $10 \mathrm{~nm}$ layer of aluminum was deposited (after optical characterization and FTIR results were obtained) to avoid charging of the sample while imaging. The SEM image of the supercell design on $11.24 \mu \mathrm{m}$ thick polyimide is shown in Figure 3a,b. The green box in Figure 3b illustrates the 4-fold symmetry of the design and, therefore, polarization-insensitivity.

Samples were optically characterized by Fourier Transform Infrared (FTIR) spectrometry, using a Bruker IFS 66v/S to obtain reflection spectra. Absorption spectra were obtained from reflection spectra by $A=1-R-T$ (see Methods). Results for all of the $5.59 \mu \mathrm{m}$ samples (narrow-band) are given in Supporting Information, note 3. The experimental absorption spectra for the $11.24 \mu \mathrm{m}$ polyimide samples are given in Figure $3 c$,d, where Figure $3 c$ corresponds to singleband fractal designs and Figure $3 \mathrm{~d}$ corresponds to the supercell design. The absorption spectra for the $11.24 \mu \mathrm{m}$ thick polyimide alone (no crosses) is shown in the dashed magenta curve (Figure 3c). Matched simulation data for single-band (individual fractal designs, 5.59 and $11.24 \mu \mathrm{m}$ ) devices are 

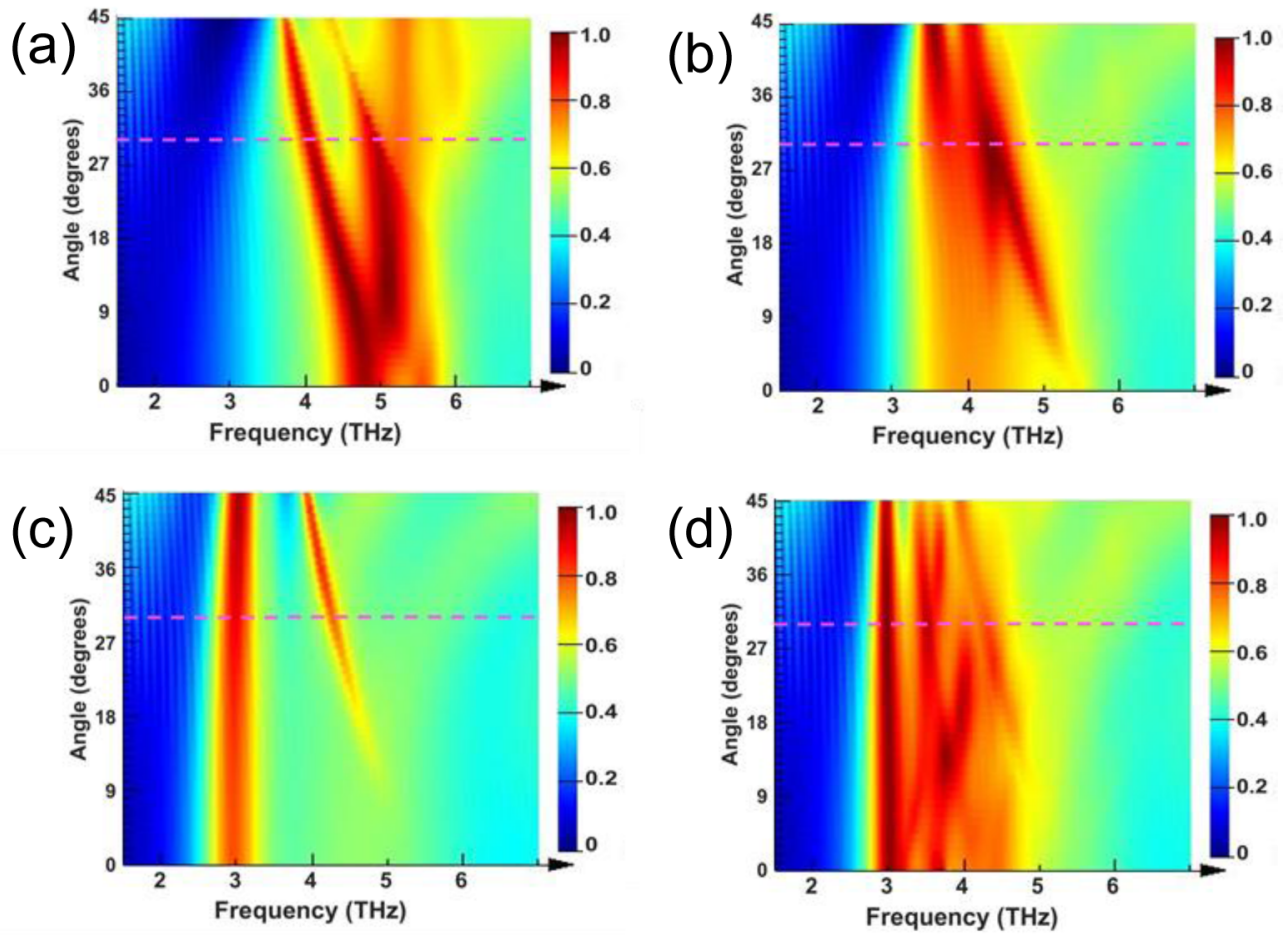

Figure 4. Numerically simulated maps of angle-dependent absorption response. Numerical simulations of the fractal cross absorbers were performed using Lumerical FDTD, where the oblique angle of incidence was varied between 0 and $45^{\circ}$. (a) Level 1 fractal design; (b) Level 2 fractal design; (c) Level 3 fractal design; (d) Broadband supercell design. The magenta dashed line is a reference to the spectral response at $30^{\circ}$ incidence, as carried out experimentally and in matched simulations. Polyimide thickness here is $11.24 \mu \mathrm{m}$.

given in Supporting Information, note 4, showing extremely good agreement.

The samples exhibit very broadband spectra, showing good agreement with preliminary simulations. However, it is clear that the absorption that takes place within the polyimide alone (Figure 3c) around $\sim 4.5 \mathrm{THz}$ modifies the spectra of the metasurface designs. We obtain superimposed broadband spectra for the single-band designs of fractal levels 1 and 2 (blue and green curves in Figure 3c) because the resonance frequencies are very near to this polyimide absorption band. However, because the resonance frequency of the level 3 fractal cross (red curve in Figure 3c) is much lower than the polyimide absorption region, we obtain two distinct features for this spectra: one peak at $2.95 \mathrm{THz}$ and another at $\sim 4.5 \mathrm{THz}$. We attribute the $\sim 4.5 \mathrm{THz}$ peak to being primarily absorption in the polyimide, while the peak at $2.95 \mathrm{THz}$ is the resonance frequency of the level 3 fractal cross itself. We therefore postulate that two absorption mechanisms take place: antireflection coating (ARC) type operation (at $\sim 4.5 \mathrm{THz}$ ) for the polyimide layer; and Salisbury Screen type operation (at $2.95 \mathrm{THz}$ for the level 3 fractal), relying on the impedance and phase modification of the light caused by the presence of the fractal crosses. These two absorption mechanisms are assumed to be general for our thick metasurface absorbers $(\sim \lambda / 4$ dielectric thickness) and can also be applied to the case for levels 1 and 2 fractal absorbers, where the spectral absorption responses are superimposed. Further explanation of this phenomena is discussed in the Supporting Information, note 5.

The experimental result for the broadband supercell metasurface, on $11.24 \mu \mathrm{m}$ polyimide, is given in Figure $3 \mathrm{~d}$ (solid black line). The spectrum shows an exceptionally broad and highly absorbing response, which agrees almost perfectly with simulated results (dashed black line); the simulation was carried out at $30^{\circ}$ incidence using the new refractive index parameters of $n=1.71+0.08 i$. Experimental peak absorption occurs at $2.95 \mathrm{THz}$ with $93 \%$ absorption. Equally significant high-absorption values occur at $3.47 \mathrm{THz}$ (92\% absorption), $3.90 \mathrm{THz}$ (88\% absorption), and $4.33 \mathrm{THz}$ (91\% absorption), with a minima of $68 \%$ at $3.18 \mathrm{THz}$. Due to the presence of this minima, we calculate the average absorption taking place over the broad bandwidth using a similar method to that carried out for the preliminary simulation in Figure 2d. Here we obtain an average experimental absorption value of $83 \%$ between the frequency range of $2.82 \mathrm{THz}$ and $5.15 \mathrm{THz}$. The averaging method is given in the Supporting Information, note 7 . Although in good agreement, we note that the differences in the experimental and simulated spectra are due to a few things. First, the fabricated samples may not be the exact dimensions as used in simulation. Also, polyimide permittivity parameters used in the simulation are nondispersive, that is, we used a single complex permittivity value to describe the full frequency range, whereas in reality it is more likely that the polyimide is dispersive with frequency.

A highly significant figure of merit of any broadband absorber is the fwhm and fractional bandwidth. Taking $93 \%$ as the maximum absorption, we have a half-maximum value of $46.5 \%$. From this, a fwhm of $\Delta f=2.82 \mathrm{THz}$ (from $2.74 \mathrm{THz}$ to 5.56 $\mathrm{THz}$ ) is obtained, which corresponds to a fractional bandwidth of $\Delta f / f_{\mathrm{c}}=68 \%$ at the central frequency of $f_{\mathrm{c}}=4.15 \mathrm{THz}$. More importantly, this fwhm bandwidth exceeds one optical octave $(5.56 \mathrm{THz} / 2.74 \mathrm{THz}=2.03)$, which is the broadest bandwidth achieved experimentally by a planar $\mathrm{THz}$ absorber to date, while simultaneously exceeding $45 \%$ absorption. This device therefore offers a distinct advantage over other broadband absorbers, in that it is planar, has sufficiently high absorption magnitudes, and covers a distinctly wide frequency bandwidth, 
paving the way toward implementation in commercial applications.

To further analyze experimental results, we performed a series of numerical simulations, using both CST MWS and Lumerical FDTD softwares. To examine the broadband characteristics of the supercell design (11.24 $\mu \mathrm{m}$ polyimide), the five prominent peaks of the matched simulation spectra (shown in Figure 3d) were investigated using 2D electric field monitors. These results are given in Supporting Information, note 8. The results show, as expected, that each peak corresponds to dominant resonances of the individual crosses. Because the experimentally obtained spectra were taken with light incident at $30^{\circ}$, while preliminary designs were carried out at normal incidence, we investigated the angular dependence of our broadband device. Shown in Figure 4 are numerically obtained absorption spectra for oblique incident angles between $0^{\circ}$ and $45^{\circ}$ for simulated $11.24 \mu \mathrm{m}$ polyimide thickness samples (the angular dependence of $5.59 \mu \mathrm{m}$ polyimide thickness samples are given in Supporting Information, note 9). This angular dependence was investigated using Lumerical FDTD software and thus differ slightly from previous simulated results (from CST) due to the difference in software solver architectures. Figure $4 a-c$ corresponds to the single-band absorbers for fractal levels 1-3, respectively, while Figure $4 \mathrm{~d}$ corresponds to the broadband supercell-absorber design. All designs seem to show a good robustness to the incident angle, with minimal change in absorption magnitude. A narrowing of the broadband performance is seen for the supercell design (Figure 4d) for angles larger than $30^{\circ}$. However, the absorption magnitude appears to be sufficiently high for all angles, and it is assumed that our supercell-based broadband absorber will perform well, albeit with a reduced bandwidth, even up to $45^{\circ}$ incident angles. Because the design is insensitive to polarization, the spectral responses will also be identical for negative angles of incidence $\left(-45^{\circ}\right.$ to $\left.+45^{\circ}\right)$.

\section{CONCLUSION}

In conclusion, we have demonstrated an ultrathin metasurface absorber capable of achieving a broad absorption bandwidth of one octave. The design relies on the combination of different fractal levels of resonant crosses, is polarization-insensitive, and achieves a peak experimental absorption magnitude of $93 \%$. We postulate a novel explanation that for a sufficiently large dielectric spacer thickness, two absorption mechanisms take place, with one being antireflection coating type absorption within the dielectric layer itself, while the second mechanism is akin to a Salisbury Screen absorber that relies on resonant structures tailoring the phase and impedance such that absorption occurs at a different frequency. The average experimental absorption is found to be $83 \%$, which is significant for a planar absorber. The octave-spanning broadband absorption exceeds $45 \%$ magnitude at the fwhm bandwidth, which is the best performing planar $\mathrm{THz}$ absorber to date. This device is highly integratable with bolometric and semiconductor detectors and is expected to provide a clear pathway to more efficient $\mathrm{THz}$ sensing devices. We have shown that using fractal resonant structures introduces an extra degree of freedom into designing broadband devices, but a downside to using fractals may occur when the fractal level is increased too much; the finite thickness of the cross arms (in our case $1 \mu \mathrm{m}$ ) will introduce a limitation on the maximum possible fractal level. At some bounded level, the fill factor will increase too much and the different parts of the fractal resonator will start to touch, causing unwanted effects and changing the resonant properties in an unknown way. One means of combatting this issue would be to decrease the arm-width, but this will introduce more complexity into fabrication if the lithography resolution is a limiting factor. In light of this, however, our device's bandwidth may be further improved with careful design of fractal levels, and is a step toward the perfect $\mathrm{THz}$ blackbody absorber.

\section{METHODS}

Numerical Simulation and Analysis. The numerical simulations were carried out in the commercially available softwares CST Microwave Studio (MWS) and Lumerical FDTD. CST MWS was used to perform Finite Element Method (FEM) calculations in the frequency domain, while Lumerical used the Finite Difference Time Domain (FDTD) solver. These softwares were used in conjunction in order to reinforce insights into the physical mechanisms taking place. Periodic boundary conditions were applied to all designs. Manual parameters were chosen for the gold conductivity and polyimide permittivity as per refs 26 and 38 . Electric field and current density plots were obtained in CST MWS, while the oblique angle of incidence plots were obtained by performing an angular sweep in Lumerical FDTD.

Fabrication. Silicon wafers of 4 in. $(10.16 \mathrm{~cm})$ diameter and $525 \mu \mathrm{m}$ thickness were first sequentially cleaned in acetone and isopropyl alcohol (IPA) in an ultrasonic bath. Following this, 10 $\mathrm{nm} \mathrm{Ti}$ and then $100 \mathrm{~nm}$ thick films of $\mathrm{Au}$ were deposited by electron beam thermal evaporation. Polyimide films were spincoated onto the wafers, where two thicknesses of polyimide were required. Using PI 2545, an $11.24 \mu$ m layer was obtained by 6 spin-coat steps, while the $5.59 \mu$ m layer was obtained using 3 spin-coat steps on separate wafers. For each layer, PI 2545 was spun at $4000 \mathrm{rpm}$ for $60 \mathrm{~s}$ and baked for $4 \mathrm{~min}$ at $140{ }^{\circ} \mathrm{C}$. After the final spin-coat and bake, the PI film was cured by hard-baking at $250{ }^{\circ} \mathrm{C}$ for $10 \mathrm{~min}$. After this, samples were cleaned in the acetone/IPA process previously mentioned, followed by an $\mathrm{O}_{2}$ plasma ashing step at $100 \mathrm{~W}$ power for 2 min (approximately $10 \mathrm{~nm} / \mathrm{min}$ PI removal rate) and then a dry etch of $\mathrm{O}_{2} / \mathrm{CF}_{4}(20 / 5 \mathrm{sccm})$ at $50 \mathrm{~W}$ for $1 \mathrm{~min}$. These steps were undertaken to aid in the adhesion of the Au fractal crosses after lift-off. A bilayer of electron beam resist was then spin-coated to define the fractal structures. First, 12\% PMMA 2010 solution was spun onto the polyimide at 5000 r.p.m. for 1 min, followed by a bake of $154{ }^{\circ} \mathrm{C}$ for $2 \mathrm{~min}$. After this, a $4 \%$ PMMA 2041 solution was spun at $5000 \mathrm{rpm}$ for $1 \mathrm{~min}$, followed by a bake of $154{ }^{\circ} \mathrm{C}$ for $2 \mathrm{~min}$. The fractal cross patterns were then defined using e-beam lithography. The complete wafer was used to prepare the varying fractal designs in order to save processing time and to inhibit the negative effects of polyimide and PMMA edge bead. After development in 1:1 IPA/MIBK (methyl isobutyl ketone) solution $\left(24^{\circ} \mathrm{C}\right.$ for $1 \mathrm{~min})$, the wafers were once again cleaned by $\mathrm{O}_{2}$ plasma ashing, using the same process as described above, to remove any organic residues in the patterned voids. Finally, $10 \mathrm{~nm}$ Ti and then $100 \mathrm{~nm}$ of $\mathrm{Au}$ was evaporated, and a lift-off process was carried out using $50{ }^{\circ} \mathrm{C}$ acetone for at least $12 \mathrm{~h}$.

Characterization of Samples. The thickness of the polyimide samples was determined by averaging the thicknesses of individual patterned samples taken from multiple areas of the processed wafer. The samples were scribed down to the silicon substrate, and the depth of the step was measured using a Dektak profilometer. The average polyimide thicknesses were 
calculated to be $5.59 \mu \mathrm{m}$ for the 3-layer wafer and $11.24 \mu \mathrm{m}$ for the 6-layer sample.

Fourier Transform Infrared (FTIR) spectrometry was carried out to obtain the absorption spectra of the samples. The equipment used to obtain the FTIR spectra was a Bruker IFS $66 \mathrm{v} / \mathrm{S}$, and the samples were under vacuum. Transmission through the samples is zero owing to the continuous $\mathrm{Au}$ ground-plane, thus only reflection measurements were required. Due to limitations of the FTIR spectrometer setup, reflection measurements could only be carried out at a $30^{\circ}$ angle. The measured reflection spectra were normalized with respect to a gold mirror using a $7 \mathrm{~mm}$ aperture. The absorption was calculated using the expression $A(\omega)=1-R(\omega)-T(\omega)$, where the transmission is zero, thus, resulting in an absorption only dependent on reflection $A(\omega)=1-R(\omega)$.

\section{ASSOCIATED CONTENT}

\section{S Supporting Information}

The Supporting Information is available free of charge on the ACS Publications website at DOI: 10.1021/acsphotonics.7b00906.

Dielectric thickness dependency of absorption spectra, average absorption of simulated broadband spectra, experimental results for $5.59 \mu \mathrm{m}$ polyimide samples, simulated spectra for single-band absorbers matched to FTIR results, theoretical postulation for two-mechanism absorption in thick absorber designs, equivalence of fractals and scaled-cross supercells, average absorption of experimental broadband spectra, 2D electric field profiles of simulated supercell design, and simulated angular dependence of $5.59 \mu \mathrm{m}$ polyimide samples (PDF).

\section{AUTHOR INFORMATION}

\section{Corresponding Authors}

*E-mail: mitchell.kenney@glasgow.ac.uk.

*E-mail: david.cumming.2@glasgow.ac.uk.

\section{ORCID}

Mitchell Kenney: 0000-0002-5009-9729

Yash D. Shah: 0000-0002-7582-7972

\section{Notes}

The authors declare no competing financial interest.

The dataset for the results in the paper can be found at http:// dx.doi.org/10.5525/gla.researchdata.482.

\section{ACKNOWLEDGMENTS}

This work was supported by the Engineering and Physical Sciences Research Council of the United Kingdom, Grant No. EP/J018678/1. Device fabrication and characterization was carried out in part at the James Watt Nanofabrication Centre (JWNC) at the University of Glasgow.

\section{REFERENCES}

(1) Woodward, R. M.; Wallace, V. P.; Pye, R. J.; Cole, B. E.; Arnone, D. D.; Linfield, E. H.; Pepper, M. Terahertz Pulse Imaging of Ex Vivo Basal Cell Carcinoma. J. Invest. Dermatol. 2003, 120, 72-78.

(2) Lee, A. W. M.; Qin, Q.; Kumar, S.; Williams, B. S.; Hu, Q.; Reno, J. L. Real-Time Terahertz Imaging over a Standoff Distance $(>25$ Meters). Appl. Phys. Lett. 2006, 89, 1-4.

(3) Baker, C.; Lo, T.; Tribe, W. R.; Cole, B. E.; Hogbin, M. R.; Kemp, M. C. Detection of Concealed Explosives at a Distance Using Terahertz Technology. Proc. IEEE 2007, 95, 1559-1565.
(4) Liu, H.; Chen, Y.; Bastiaans, G. J.; Zhang, X.-C. Detection and Identification of Explosive RDX by THz Diffuse Reflection Spectroscopy. Opt. Express 2006, 14, 415.

(5) Federici, J.; Moeller, L. Review of Terahertz and Subterahertz Wireless Communications. J. Appl. Phys. 2010, 107, 111101.

(6) Strachan, C. J.; Taday, P. F.; Newnham, D. A.; Gordon, K. C.; Zeitler, J. A.; Pepper, M.; Rades, T. Using Terahertz Pulsed Spectroscopy to Quantify Pharmaceutical Polymorphism and Crystallinity. J. Pharm. Sci. 2005, 94, 837-846.

(7) Chen, X.; Huang, L.; Mühlenbernd, H.; Li, G.; Bai, B.; Tan, Q.; Jin, G.; Qiu, C.-W.; Zhang, S.; Zentgraf, T. Dual-Polarity Plasmonic Metalens for Visible Light. Nat. Commun. 2012, 3, 1198.

(8) Wen, D.; Yue, F.; Ardron, M.; Chen, X. Multifunctional Metasurface Lens for Imaging and Fourier Transform. Sci. Rep. 2016, 6, 27628.

(9) Khorasaninejad, M.; Chen, W. T.; Devlin, R. C.; Oh, J.; Zhu, A. Y.; Capasso, F. Metalenses at Visible Wavelengths: Diffraction-Limited Focusing and Subwavelength Resolution Imaging. Science 2016, 352, $1190-1194$.

(10) Huang, L.; Chen, X.; Mühlenbernd, H.; Zhang, H.; Chen, S.; Bai, B.; Tan, Q.; Jin, G.; Cheah, K.-W.; Qiu, C.-W.; et al. ThreeDimensional Optical Holography Using a Plasmonic Metasurface. Nat. Commun. 2013, 4, 2808.

(11) Zheng, G.; Mühlenbernd, H.; Kenney, M.; Li, G.; Zentgraf, T.; Zhang, S. Metasurface Holograms Reaching $80 \%$ Efficiency. Nat. Nanotechnol. 2015, 10, 308-312.

(12) Yu, N.; Genevet, P.; Kats, M. a.; Aieta, F.; Tetienne, J.-P.; Capasso, F.; Gaburro, Z. Light Propagation with Phase Discontinuities: Generalized Laws of Reflection and Refraction. Science 2011, 334, 333-337.

(13) Liu, L.; Zhang, X.; Kenney, M.; Su, X.; Xu, N.; Ouyang, C.; Shi, Y.; Han, J.; Zhang, W.; Zhang, S. Broadband Metasurfaces with Simultaneous Control of Phase and Amplitude. Adv. Mater. 2014, 26, 5031-5036.

(14) Plum, E.; Fedotov, V. A.; Zheludev, N. I. Asymmetric Transmission: A Generic Property of Two-Dimensional Periodic Patterns. J. Opt. 2011, 13, 024006.

(15) Kenney, M.; Li, S.; Zhang, X.; Su, X.; Kim, T.; Wang, D.; Wu, D.; Ouyang, C.; Han, J.; Zhang, W.; et al. Pancharatnam-Berry Phase Induced Spin-Selective Transmission in Herringbone Dielectric Metamaterials. Adv. Mater. 2016, 28, 9567-9572.

(16) Khorasaninejad, M.; Chen, W. T.; Zhu, A. Y.; Oh, J.; Devlin, R. C.; Rousso, D.; Capasso, F. Multispectral Chiral Imaging with a Metalens. Nano Lett. 2016, 16, 4595-4600.

(17) Valentine, J.; Li, J.; Zentgraf, T.; Bartal, G.; Zhang, X. An Optical Cloak Made of Dielectrics. Nat. Mater. 2009, 8, 568-571.

(18) Ergin, T.; Stenger, N.; Brenner, P.; Pendry, J. B.; Wegener, M. Three-Dimensional Invisibility Cloak at Optical Wavelengths. Science 2010, 328, 337-339.

(19) Ni, X.; Wong, Z. J.; Mrejen, M.; Wang, Y.; Zhang, X. An Ultrathin Invisibility Skin Cloak for Visible Light. Science 2015, 349, $1310-1314$.

(20) Landy, N. I.; Sajuyigbe, S.; Mock, J. J.; Smith, D. R.; Padilla, W. J. Perfect Metamaterial Absorber. Phys. Rev. Lett. 2008, 100, 207402.

(21) Liu, N.; Mesch, M.; Weiss, T.; Hentschel, M.; Giessen, H. Infrared Perfect Absorber and Its Application as Plasmonic Sensor. Nano Lett. 2010, 10, 2342-2348.

(22) Hao, J.; Wang, J.; Liu, X.; Padilla, W. J.; Zhou, L.; Qiu, M. High Performance Optical Absorber Based on a Plasmonic Metamaterial. Appl. Phys. Lett. 2010, 96, 251104.

(23) Huang, L.; Chen, H. A Brief Review on Terahertz Metamaterial Perfect Absorbers. Terahertz Sci. Technol. 2013, 6, 26-39.

(24) Salisbury, W. W. Absorbent Body for Electromagnetic Waves. US2599944, 1952.

(25) Munk, B. A. Frequency Selective Surfaces: Theory and Design; John Wiley \& Sons, 2005.

(26) Grant, J.; Ma, Y.; Saha, S.; Khalid, A.; Cumming, D. R. S. Polarization Insensitive, Broadband Terahertz Metamaterial Absorber. Opt. Lett. 2011, 36, 3476-3478. 
(27) Cui, Y.; Fung, K. H.; Xu, J.; Ma, H.; Jin, Y.; He, S.; Fang, N. X. Ultrabroadband Light Absorption by a Sawtooth Anisotropic Metamaterial Slab. Nano Lett. 2012, 12, 1443-1447.

(28) Shen, X.; Yang, Y.; Zang, Y.; Gu, J.; Han, J.; Zhang, W.; Jun Cui, T. Triple-Band Terahertz Metamaterial Absorber: Design, Experiment, and Physical Interpretation. Appl. Phys. Lett. 2012, 101, 154102.

(29) Zhu, J.; Ma, Z.; Sun, W.; Ding, F.; He, Q.; Zhou, L.; Ma, Y. Ultra-Broadband Terahertz Metamaterial Absorber. Appl. Phys. Lett.

2014, 105, 21102.

(30) Li, X.; Liu, H.; Sun, Q.; Huang, N. Ultra-Broadband and Polarization-Insensitive Wide-Angle Terahertz Metamaterial Absorber. Photonics Nanostructures - Fundam. Appl. 2015, 15, 81-88.

(31) Liu, S.; Chen, H.; Cui, T. J. A Broadband Terahertz Absorber Using Multi-Layer Stacked Bars. Appl. Phys. Lett. 2015, 106, 151601.

(32) Liu, S.; Zhuge, J.; Ma, S.; Chen, H.; Bao, D.; He, Q.; Zhou, L.; Cui, T. J. A Bi-Layered Quad-Band Metamaterial Absorber at Terahertz Frequencies. J. Appl. Phys. 2015, 118, 245304.

(33) Ma, Y.; Chen, Q.; Grant, J.; Saha, S. C.; Khalid, a; Cumming, D. R. S. A Terahertz Polarization Insensitive Dual Band Metamaterial Absorber. Opt. Lett. 2011, 36, 945-947.

(34) Grant, J. P.; McCrindle, I. J. H.; Cumming, D. R. S. Simulation, Fabrication and Characterization of $\mathrm{THz}$ Metamaterial Absorbers. J. Visualized Exp. 2012, e50114-e50114.

(35) Miyamaru, F.; Saito, Y.; Takeda, M. W.; Hou, B.; Liu, L.; Wen, W.; Sheng, P. Terahertz Electric Response of Fractal Metamaterial Structures. Phys. Rev. B: Condens. Matter Mater. Phys. 2008, 77, 45124.

(36) Kubota, S.; Miyamaru, F.; Takeda, M. W. Terahertz Response of Fractal Metamaterials. 2009 34th Int. Conf. on Infrared, Millimeter, and Terahertz Waves; IEEE, 2009, pp 1-2.

(37) Krzysztofik, W. J. Fractal Geometry in Electromagnetics Applications - from Antenna to Metamaterials. Microw. Rev. 2013, $19,3-14$.

(38) Tang, S.; He, Q.; Xiao, S.; Huang, X.; Zhou, L. Fractal Plasmonic Metamaterials: Physics and Applications. Nanotechnol. Rev. 2015, 4, na.

(39) Liao, Z.; Gong, R.; Nie, Y.; Wang, T.; Wang, X. Absorption Enhancement of Fractal Frequency Selective Surface Absorbers by Using Microwave Absorbing Material Based Substrates. Photonics Nanostructures - Fundam. Appl. 2011, 9, 287-294.

(40) Wang, Y.; Sun, T.; Paudel, T.; Zhang, Y.; Ren, Z.; Kempa, K. Metamaterial-Plasmonic Absorber Structure for High Efficiency Amorphous Silicon Solar Cells. Nano Lett. 2012, 12, 440-445.

(41) Aslan, E.; Korkmaz, S.; Kaya, S.; Turkmen, M. Rotated First Iteration Square Fractal Shaped Perfect Absorbers. Opt. Sensors, Sensors 2015, 2015, 316.

(42) Padilla, W. J.; Aronsson, M. T.; Highstrete, C.; Lee, M.; Taylor, a. J.; Averitt, R. D. Electrically Resonant Terahertz Metamaterials: Theoretical and Experimental Investigations. Phys. Rev. B: Condens. Matter Mater. Phys. 2007, 75, 1-4.

(43) Tao, H.; Bingham, C. M.; Strikwerda, A. C.; Pilon, D.; Shrekenhamer, D.; Landy, N. I.; Fan, K.; Zhang, X.; Padilla, W. J.; Averitt, R. D. Highly Flexible Wide Angle of Incidence Terahertz Metamaterial Absorber: Design, Fabrication, and Characterization. Phys. Rev. B: Condens. Matter Mater. Phys. 2008, 78, 2-5. 